\title{
BfDI: Mindestanforderungen an die Stellung der behördlichen Datenschutzbeauftragten in der Bundesverwaltung
}

Effektiver Datenschutz erfordert nicht nur unabhängige und funktionsfähige Datenschutzbehörden, sondern auch handlungsfähige behördliche Datenschutzbeauftragte.

Die behördlichen und betrieblichen Datenschutzbeauftragten sind eine feste Institution des deutschen Datenschutzsystems und aus diesem nicht wegzudenken. Als hausinterne Datenschutzexperten wirken sie frühzeitig in den Behörden und Unternehmen auf die Einhaltung des Datenschutzes hin.

$\mathrm{Zu}$ diesem Zweck beraten sie die Leiter der verantwortlichen Stellen und die Beschäftigten, sie kontrollieren die Einhaltung der datenschutzrechtlichen Vorschriften, schulen das Personal, fungieren als Ansprechpartner für die Betroffenen und führen die Vorabkontrollen durch - ein breites Aufgabenspektrum, das zugleich wesentlich zur Entlastung der Datenschutzbehörden beiträgt.

Das Bundesdatenschutzgesetz sieht eine Reihe von Strukturprinzipien vor, um eine sachgerechte Ausübung der Funktion zu gewährleisten. Prägend für das gesetzliche Leitbild ist die Unabhängigkeit als unabdingbare Voraussetzung für eine Amtsausübung frei von äußerer Einflussnahme.

In Ausübung ihrer Fachkunde unterliegen die Datenschutzbeauftragten keinen Weisungen. Es besteht ein umfassendes Benachteiligungsverbot und - ganz im Gegenteil - eine aktive Unterstützungspflicht durch die Dienststelle: Durch eine sachgerechte Zuweisung von Sach- und Personalmitteln, die Freistellung von sonstigen Aufgaben und die Möglichkeit zur Teilnahme an Fortbildungen auf Kosten der Arbeitsstelle. Datenschutzbeauftragte genießen zudem einen besonderen Widerrufs- und Kündigungsschutz. Die unmittelbare Unterstellung unter die Hausleitung und ein direktes Vorspracherecht - Einschätzungen der Datenschutzbeauftragten sollen nicht durch Vorgesetzte „vorgefiltert" werden - sichern die Unabhängigkeit auch in organisatorischer Hinsicht ab.

Das Gesetz zeichnet ein klares Bild, das die Datenschutzbeauftragten zu einer unabhängigen und selbstbewussten Amtswahr- nehmung befähigen soll. In der Praxis sind die Arbeitsbedingungen der Datenschutzbeauftragten allerdings höchst unterschiedlich und bisweilen defizitär ausgestaltet - ein Befund, der sicherlich nicht nur für die Bundesverwaltung gilt.

Erfahrungsgemäß zählen Aspekte der Weisungsfreiheit, der Personal- und Sachausstattung einschließlich der zeitlichen Freistellung von anderen Aufgaben, der Sicherstellung einer frühzeitigen Einbindung und Beteiligung in Vorhaben mit Datenschutzbezug, des Schutzes vor direkten oder indirekten Nachteilen und der unmittelbaren Unterstellung unter die Hausleitung zu den häufig auftretenden Praxisproblemen.

Die im April 2015 veröffentlichen Mindestanforderungen der Bundesbeauftragten für den Datenschutz und die Informationsfreiheit an die Organisation und Arbeitsweise geben nun erstmals einheitliche Standards für die Stellung der behördlichen Datenschutzbeauftragten in der Bundesverwaltung vor. Sie sollen die Datenschutzbeauftragten in ihrem Amt stärken und den öffentlichen Stellen des Bundes Hilfestellung bei der Umsetzung der gesetzlichen Anforderungen an die organisatorische Stellung und Aufgabenwahrnehmung der Datenschutzbeauftragten geben. Die Unabhängigkeit und Weisungsfreiheit, die Unterstützungspflicht durch die Dienststelle und der Schutz vor Benachteiligungen sind unabdingbare Strukturbedingungen der Funktion. Diese Aspekte werden in den Mindestanforderungen anhand der gesetzlichen Vorgaben präzisiert.

Die Mindestanforderungen der BfDI ergänzen den Beschluss des Düsseldorfer Kreises zu den Anforderungen an die Fachkunde und Unabhängigkeit der Datenschutzbeauftragten vom November 2010 und sind abrufbar unter www.bfdi.bund.de.

Andrea Voßhoff

Bundesbeauftragte für den Datenschutz und die Informationsfreiheit 